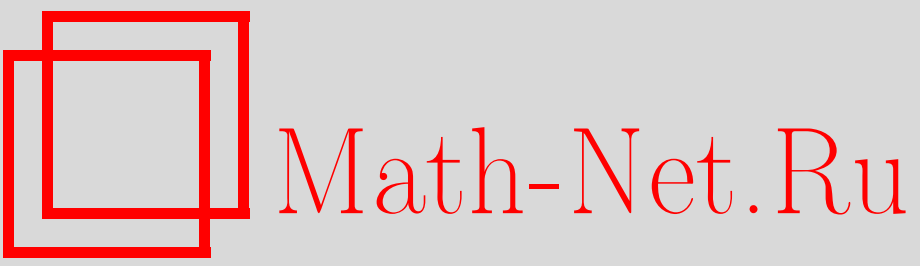

А. А. Шваб, Метод граничных интегральных уравнений для голоморфного вектора для решения задач мониторинга упругих статических полей в действующих объектах, Вестн. Сам. гос. техн. ун-та. Сер. Физ.-мат. науки, 2010, выпуск 1(), 96-103

DOI: https://doi.org/10.14498/vsgtu747

Использование Общероссийского математического портала Math-Net.Ru подразумевает, что вы прочитали и согласны с пользовательским соглашением

http://www. mathnet.ru/rus/agreement

Параметры загрузки:

IP : 54.237 .206 .68

26 апреля 2023 г., 15:55:50 
УДК 539.3

\title{
МЕТОД ГРАНИЧНЫХ ИНТЕГРАЛЬНЫХ УРАВНЕНИЙ ДЛЯ ГОЛОМОРФНОГО ВЕКТОРА ДЛЯ РЕШЕНИЯ ЗАДАЧ МОНИТОРИНГА УПРУГИХ СТАТИЧЕСКИХ ПОЛЕЙ В ДЕЙСТВУЮЩИХ ОБЪЕКТАХ
}

\author{
А. А. Шваб \\ Институт гидродинамики им. М.А. Лаврентьева СО РАН, \\ 630090, Новосибирск, пр-т Академика Лаврентьева, 15. \\ E-mail: schwab@ngs.ru
}

Рассматриваются неклассические задачи теории упругости определения или оценки изменений напряжённо-дебормированного состояния действующих объектов по натурным замерам на их границах. Полагается, что на части границы известны одновременно векторы нагрузки и перемещений, на других частях гранищы могут быть заданы отдельно вектор нагрузки или вектор перемещения, а на осталъной части поверхности (ненулевой меръ) условия не определены. Показано, что решение этих задач можно свести к решению систем граничных интегральных уравнений для голоморфного вектора.

Ключевые слова: граничные интегральные уравнения, неклассические задачи упругости, задачи мониторинга, упругие поля.

Рассматривается пространственная задача статической теории упругости для однородного изотропного тела, когда условия заданы на трех частях поверхности тела, а на четвертой части с ненулевой мерой условия не определены. При этом полагаем, что на одной части заданы одновременно вектор перемещений $\boldsymbol{u}$, вектор нагрузки $\boldsymbol{p}$, а на двух других частях - произвольная комбинация векторов перемещений и нагрузки. Подобные задачи связаны с проблемой мониторинга действующих объектов, когда необходимо оценить напряжённо-деформированное состояние или его флуктуации в процессе эксплуатации по натурным или экспериментальным замерам. Так, например, если на свободной поверхности тела $(\boldsymbol{p}=\mathbf{0})$ измерен вектор перемещений $\boldsymbol{u}$, то на этой части поверхности заданы переопределенные условия в смысле классических постановок задач теории упругости.

Например, по изменениям вектора перемещений на дневной поверхности Земли необходимо оценить изменения тектонических напряжений в земной коре [1]. K этому классу задач также можно отнести задачи дефектоскопии на основе метода голографической интерферометрии, задачи тензометрии и тензочувствительных покрытий, задачи об оценке напряжённо-деформированного состояния подземных сооружений по натурным замерам и ряд других обратных задач. В дальнейшем, если такие данные известны только на одной части поверхности, а на остальной части условия не определены, то согласно $[2,3]$ такую задачу будем называть задачей $(\boldsymbol{u}, \boldsymbol{p})$.

Задача $(\boldsymbol{u}, \boldsymbol{p})$ относится к условно-корректным задачам [3]. В работах [4-10] исследуются различные подходы к решению этой задачи. В основном методы решения базируются на методе граничных интегральных уравнениях

Алъберт Александрович Шваб (д.ф.-м.н.), ведущий научный сотрудник, лаб. статической прочности. 
и методах регуляризации. По причине численной неустойчивости в основном рассмотрены только двумерные задачи.

Чисто поверхность, на которой могут быть заданы переопределённые данные, может быть существенно меньше поверхности всего тела. В этом случае, в силу численной неустойчивости задачи и приближённого характера начальных данных, прямые методы решения задачи $(\boldsymbol{u}, \boldsymbol{p})$ неэффективны. Решение в этом случае строится путем перебора различных решений классических краевых задач, минимизирующих ошибки на переопределенных данных [4-9]. Недостатком такого подхода является выбор адекватного класса решения для функционала минимизации, поскольку, имея ошибки одного порядка, классы решений могут отличаться значительно.

Одним из подходов к решению этой проблемы может быть привлечение дополнительной информации об объекте, чтобы с достаточной точностью восстановить напряжённо-деформированное состояние объекта: можно использовать некоторые данные об объекте при его проектировании; в процессе эксплуатации объекта определённые граничные условия, а именно значения перемещений $\boldsymbol{u}$ или нагрузок $\boldsymbol{p}$ могут считаться неизменными, т. е. совпадающими с проектными, и т. д. Таким образом, экспериментальные данные и информация о проектировании объекта приводят к новой неклассической задаче теории упругости: необходимо восстановить напряжённо-деформированное состояние в теле, когда на одной части поверхности тела заданы условия $(\boldsymbol{u}, \boldsymbol{p})$, на двух других частях - условия для $\boldsymbol{u}$ или $\boldsymbol{p}$, а на оставшейся части поверхности (ненулевой меры) условия не определены. Подобные задачи нельзя отнести ни к задаче Коши, ни к классическим краевым задачам теории упругости.

Для решения неклассических пространственных задач в $[10,14]$ предложено использовать представление общего решения задачи теории упругости через голоморфный вектор.

В этой работе рассматриваются решения выделенных выше неклассических задач теории упругости через граничные интегральные уравнения для голоморфного вектора. Сформулируем основные неклассические задачи.

ЗАДАЧА І (ЗАДАЧА $(\boldsymbol{u}, \boldsymbol{p}))$. Пусть однородное Изотропное упругое тело оббёма $\Omega$ с поверхностью $\partial \Omega$ находится под действием определённых нагрузок. На части поверхности $S_{u \sigma} \subset \partial \Omega$ известнь

$$
u_{j}=u_{j}(\boldsymbol{r}), \quad p_{j}=\sigma_{i j}(\boldsymbol{r}) n_{i}(\boldsymbol{r}) \quad(i, j \equiv 1,2,3), \quad \boldsymbol{r} \in S_{u \sigma} .
$$

Необходимо по этим данным восстановить значения вектора и в заданном теле.

ЗАДАчА II. Пусть в однородном изотропном упругом теле обгёма $\Omega$ с поверхностью $\partial \Omega$ кроме условий задачи I на частях поверхности $S_{u} \subset \partial \Omega$ и $S_{p} \subset \partial \Omega$ заданы дополнительные условия, т.е. на поверхности тела $\partial \Omega$ имеем

$$
\begin{gathered}
u_{j}=u_{j}(\boldsymbol{r}), \quad p_{j}=\sigma_{i j}(\boldsymbol{r}) n_{i}(\boldsymbol{r}) \quad(i, j \equiv 1,2,3), \quad \boldsymbol{r} \in S_{u \sigma} ; \\
p_{j}=\sigma_{i j}(\boldsymbol{r}) n_{i}(\boldsymbol{r}) \quad(i, j \equiv 1,2,3), \quad \boldsymbol{r} \in S_{p} ; \\
u_{j}=u_{j}(\boldsymbol{r}) \quad(j \equiv 1,2,3), \quad \boldsymbol{r} \in S_{u} ; \quad S_{u} \cup S_{p} \cup S_{u \sigma} \neq \partial \Omega .
\end{gathered}
$$

Необходимо по этим данным восстановить значения вектора и в заданном теле. 
Построим решение задачи $(\boldsymbol{u}, \boldsymbol{p})$, т. е. когда граничные значения заданы только на части $S_{u \sigma}$ поверхности тела $\partial \Omega$.

Заметим, что согласно [3] условия $(\boldsymbol{u}, \boldsymbol{p})$ на поверхности $S_{u \sigma}$ позволяют найти все значения $\frac{\partial u_{i}}{\partial x_{j}}$ на $S_{u \sigma}$. Поэтому на $S_{u \sigma}$ могут быть восстановлены значения $\operatorname{div} \boldsymbol{u}$ и $\operatorname{rot} \boldsymbol{u}$ вектора $\boldsymbol{u}$.

Приведём необходимые определения и выводы. Пусть $\boldsymbol{u}$ - вектор упругого перемещения, т. е. $\boldsymbol{u}$ - решение уравнения Ламе:

$$
L \boldsymbol{u} \equiv(\lambda+2 \mu) \operatorname{grad}(\operatorname{div} \boldsymbol{u})-\mu \operatorname{rot}(\operatorname{rot} \boldsymbol{u})=0 .
$$

Введём следующие скалярную и векторную функции $f_{0}(\boldsymbol{r})=(\lambda+2 \mu) \operatorname{div} \boldsymbol{u}$, $\boldsymbol{e}(\boldsymbol{r})=-\mu \operatorname{rot} \boldsymbol{u}$. Представим (1) в виде

$$
\begin{aligned}
& \operatorname{grad} \boldsymbol{e}(\boldsymbol{r})=0, \\
& \operatorname{grad} f_{0}(\boldsymbol{r})+\operatorname{rot} \boldsymbol{e}(\boldsymbol{r})=0 .
\end{aligned}
$$

Система (2) является системой Моисила-Теодореску [12]. Кватернионная функция $\boldsymbol{F}(\boldsymbol{r})=f_{0}(\boldsymbol{r})+\boldsymbol{e}(\boldsymbol{r})$ представляет собой леворегулярную функцию кватернионной переменной [11]. А. В. Бицадзе, изучая решение системы Моисила-Теодореску, назвал четырёхкомпонентный вектор $\boldsymbol{F}(\boldsymbol{r})=\left\{f_{0}, e_{1}, e_{2}, e_{3}\right\}$, удовлетворяющий (2), голоморфным вектором [13]. В данном случае $e_{i}=$ $=-\mu(\operatorname{rot} \boldsymbol{u})_{i}(i \equiv 1,2,3)$. Для голоморфного вектора доказаны теоремы, аналогичные теоремам в теории функции комплексного переменного. Приведём основную теорему для голоморфного вектора.

Теорема. Если вектор $\boldsymbol{F}(\boldsymbol{r}) \in C^{1}$ в области $\Omega^{+} \subset E_{3}$ с кусочно-гладкой границей по Ляпунову удовлетворяет системе Моисила-Теодореску в $\Omega^{+}$, mo

$$
\begin{aligned}
& \iint_{\partial \Omega} D\left(n_{1}, n_{2}, n_{3}\right) \boldsymbol{F}(\boldsymbol{r}) d S_{\boldsymbol{r}}=0, \\
& \frac{1}{4 \pi} \iint_{\partial \Omega} \boldsymbol{M}\left(\left|\boldsymbol{r}-\boldsymbol{r}_{0}\right|\right) \boldsymbol{F}\left(\boldsymbol{r}_{0}\right) d S_{\boldsymbol{r}_{0}}=\Lambda(\boldsymbol{r}),
\end{aligned}
$$

где $\boldsymbol{r}=\left\{X_{1}, X_{2}, X_{3}\right\}, \boldsymbol{r}_{0}=\left\{Y_{1}, Y_{2}, Y_{3}\right\}$,

$$
\Lambda(r)=\left\{\begin{array}{cc}
\boldsymbol{F}(\boldsymbol{r}), & \boldsymbol{r} \in \Omega^{+}, \\
0, & \boldsymbol{r} \in \Omega^{-},
\end{array}\right.
$$

$\boldsymbol{n}=\left\{n_{1}, n_{2}, n_{3}\right\}-$ вектор нормали к поверхности $\partial \Omega$ области $\Omega^{+}$, $\boldsymbol{M}\left(\left|\boldsymbol{r}-\boldsymbol{r}_{0}\right|\right)$ - матричный дифференииальный оператор:

$$
\begin{gathered}
M\left(\left|\boldsymbol{r}-\boldsymbol{r}_{0}\right|\right)=-D^{*}\left(\frac{\partial}{\partial Y_{1}}, \frac{\partial}{\partial Y_{2}}, \frac{\partial}{\partial Y_{3}}\right) \frac{1}{\left|\boldsymbol{r}-\boldsymbol{r}_{0}\right|} D\left(n_{1}, n_{2}, n_{3}\right), \\
D\left(C_{1}, C_{2}, C_{3}\right)=\left\|\begin{array}{cccr}
0 & C_{1} & C_{2} & C_{3} \\
C_{1} & 0 & -C_{3} & C_{2} \\
C_{2} & C_{3} & 0 & -C_{2} \\
C_{3} & -C_{2} & C_{1} & 0
\end{array}\right\|
\end{gathered}
$$


Равенство (3) представляет собой аналог интегральной теоремы Коши, а (4) - интегральной формулы Коши. Кроме перечисленных свойств для векторов $\boldsymbol{F}(\boldsymbol{r})$ установлены аналоги теоремы Морера, формулы Сохоцкого-Племели, принцип Римана-Шварца и ряд других утверждений. Заметим, что соотношение (4) при $\boldsymbol{r} \in \Omega^{-}$можно рассматривать как теорему о граничных значениях голоморфного вектора, т. е. если для четырёхкомпонентного вектора $\boldsymbol{F}(\boldsymbol{r})$ выполнено условие (4) при $\boldsymbol{r} \in \Omega^{-}$, то $\boldsymbol{F}(\boldsymbol{r})$ на $\partial \Omega$ есть граничные значения голоморфного вектора в $\Omega$. В [14] установлены следующие утверждения.

УтвеРждение 1. Задача $(\boldsymbol{u}, \boldsymbol{p})$ может быть сведена $к$ задаче восстановления голоморфного вектора в $\Omega$ по его значениям на части поверхности $S_{u \sigma}$.

УтвеРЖДЕниЕ 2. Справедлива теорема единственности голоморфного продолжения.

Отметим два основных пути численной реализации задачи $(\boldsymbol{u}, \boldsymbol{p})$. Первый путь основан на решении задачи Коши для системы Моисила-Теодореску конечно-разностным методом [14]. Второй - на приведении задачи к интегральному уравнению. Так, из интегральной формулы Коши для нахождения голоморфного вектора $\boldsymbol{F}(\boldsymbol{r})$ по его значениям $\boldsymbol{F}^{*}(\boldsymbol{r})$ на части поверхности $S_{u \sigma} \subset \partial \Omega$ получим

$$
-\frac{1}{2} \boldsymbol{F}(\boldsymbol{r})+\frac{1}{4 \pi} \iint_{\partial \Omega \backslash S_{u \sigma}} \boldsymbol{M}\left(\left|\boldsymbol{r}-\boldsymbol{r}_{0}\right|\right) \boldsymbol{F}\left(\boldsymbol{r}_{0}\right) d S_{\boldsymbol{r}_{0}}=\boldsymbol{Q}^{*}(\boldsymbol{r}),
$$

где

$$
\boldsymbol{Q}^{*}(\boldsymbol{r})=-\frac{1}{4 \pi} \iint_{S_{u \sigma}} \boldsymbol{M}\left(\left|\boldsymbol{r}-\boldsymbol{r}_{0}\right|\right) \boldsymbol{F}^{*}\left(\boldsymbol{r}_{0}\right) d S_{r_{0}} .
$$

Таким образом, решение системы Моисила-Теодореску может быть сведено к интегральному уравнению (5) для голоморфного вектора. Отметим, что уравнение (5) при $\boldsymbol{r} \in \partial \Omega \backslash S_{u \sigma}$ - сингулярное, а при $\boldsymbol{r} \in S_{u \sigma}$ - первого рода. Из решения уравнения (5) при $\boldsymbol{r} \in \partial \Omega \backslash S_{u \sigma}$ определяется голоморфный вектор $\boldsymbol{F}(\boldsymbol{r})$, затем из (4) - значение $\boldsymbol{F}(\boldsymbol{r})$ в $\Omega^{+}$.

После нахождения голоморфного вектора $\boldsymbol{F}(\boldsymbol{r})=f_{0}(\boldsymbol{r})+\boldsymbol{e}(\boldsymbol{r})$ определяются значения $\operatorname{div} \boldsymbol{u}=f_{0}(\boldsymbol{r}) /(\lambda+2 \mu)$ и $\operatorname{rot} \boldsymbol{u}=-\boldsymbol{e}(\boldsymbol{r}) / \mu$ в $\Omega$.

На следующем этапе решения задачи $(\boldsymbol{u}, \boldsymbol{p})$ формулируется новая задача об отыскании вектора перемещений по известным значениям $\operatorname{div} \boldsymbol{u}, \operatorname{rot} \boldsymbol{u}$ в $\Omega$ и граничным условиям на $S_{u \sigma}$.

Построим вектор $\boldsymbol{A}(\boldsymbol{r})$ такой, что

$$
\operatorname{div} \boldsymbol{A}=f_{0}(\boldsymbol{r}) /(\lambda+2 \mu), \quad \operatorname{rot} \boldsymbol{A}=-\boldsymbol{e}(\boldsymbol{r}) / \mu .
$$

Нахождение вектора $\boldsymbol{A}$ можно представить как нахождение суммы двух векторов $\boldsymbol{A}_{1}$ и $\boldsymbol{A}_{2}$ таких, что вектор $\boldsymbol{A}_{1}$ определяется из следующих уравнений:

$$
\operatorname{rot} \boldsymbol{A}_{1}=0, \quad \operatorname{div} \boldsymbol{A}_{1}=f_{0}(\boldsymbol{r}) /(\lambda+2 \mu),
$$

а вектор $\boldsymbol{A}_{2}$ находится из соотношений

$$
\operatorname{rot} \boldsymbol{A}_{2}=-\boldsymbol{e}(\boldsymbol{r}) / \mu, \quad \operatorname{div} \boldsymbol{A}_{2}=0 .
$$


В этом случае вектор $\boldsymbol{A}_{1}$ можно представить в виде $\boldsymbol{A}_{1}=\operatorname{grad} C(\boldsymbol{r})$, где

$$
C(\boldsymbol{r})=-\frac{1}{4 \pi} \iiint_{\Omega} \frac{f_{0}(\boldsymbol{r})}{(\lambda+2 \mu)\left|\boldsymbol{r}-\boldsymbol{r}_{0}\right|} d V_{\boldsymbol{r}_{0}}
$$

а вектор $\boldsymbol{A}_{2}$ - в виде $\boldsymbol{A}_{2}=\operatorname{rot} \boldsymbol{\Psi}$ и $\operatorname{div} \boldsymbol{\Psi}=0$. Тогда из равенств $\operatorname{rot} \boldsymbol{A}_{2}=$ $=\operatorname{rot}(\operatorname{rot} \boldsymbol{\Psi})=\operatorname{div}(\operatorname{grad} \boldsymbol{\Psi})-\Delta \boldsymbol{\Psi}$ можно записать, что $\Delta \boldsymbol{\Psi}=\boldsymbol{e}(\boldsymbol{r}) / \mu$.

Для определения вектор функции $\boldsymbol{\Psi}(\boldsymbol{r})$ строим частное решение в виде

$$
\boldsymbol{\Psi}(\boldsymbol{r})=-\frac{1}{4 \pi \mu} \iiint_{\Omega} \frac{\boldsymbol{e}(\boldsymbol{r})}{\left|\boldsymbol{r}-\boldsymbol{r}_{0}\right|} d V_{\boldsymbol{r}_{0}} .
$$

Искомый вектор перемещения $\boldsymbol{u}$ отличается от вектора $\boldsymbol{A}$ на потенциальный вектор $\boldsymbol{b}$ такой, что $\operatorname{div} \boldsymbol{b}=\operatorname{rot} \boldsymbol{b}=0, \boldsymbol{b}=\boldsymbol{u}-\boldsymbol{A}$. Для потенциального вектора $\boldsymbol{b}=\left\{b_{1}, b_{2}, b_{3}\right\}$ на $S_{u \sigma}$ можно поставить в соответствие голоморфный вектор $\boldsymbol{B}(\boldsymbol{r})=\left\{0, b_{1}, b_{2}, b_{3}\right\}$. Поэтому задача определения потенциального вектора $\boldsymbol{b}$ может быть также сведена к (5), но уже для отыскания голоморфного вектора $\boldsymbol{B}(\boldsymbol{r})=\left\{0, b_{1}, b_{2}, b_{3}\right\}$ в $\Omega$ с граничными условиями $\boldsymbol{B}(\boldsymbol{r})=\left\{0, u_{1}-A_{1}\right.$, $\left.u_{2}-A_{2}, u_{3}-A_{3}\right\}$ на $S_{u \sigma}$. Окончательно решение в $\Omega$ представим как $\boldsymbol{u}=\boldsymbol{a}+\boldsymbol{b}$. Это и есть полная цепь решения задачи $(\boldsymbol{u}, \boldsymbol{p})$.

Рассмотрим задачу II. Запишем условия на $\partial \Omega$ для $f_{0}(\boldsymbol{r})$ и $\boldsymbol{e}(\boldsymbol{r})$. Так, для компоненты $f_{0}(\boldsymbol{r})=(\lambda+2 \mu) \operatorname{div} \boldsymbol{u}$ из закона Гука и соотношения Коши на $S_{u \sigma}$ получим

$$
f_{0}(\boldsymbol{r})=p_{3}+2 \mu\left\{\sum_{i=1}^{2}\left(\frac{\partial}{\partial a_{i}}\left[\frac{u_{i}}{H_{i}}\right]+\frac{1}{2 H_{i}^{2}} \sum_{j=1}^{3} \frac{\partial H_{i}}{\partial a_{j}} \frac{u_{j}}{H_{j}}\right)\right\} .
$$

Выражение в фигурных скобках, умноженное на 2 , обозначим через $N_{1}$. Здесь $a_{i}$ - криволинейные ортогональные координаты, связанные с поверхностью $\partial \Omega$. Так, $a_{1}, a_{2}$-координаты по касательной, а $a_{3}$-по нормали к поверхности; $H_{i}-$ коэффициенты Ламе поверхности $\partial \Omega$. Обозначим $\boldsymbol{\omega}=\frac{1}{2}(\operatorname{rot} \boldsymbol{u})$, тогда, используя соотношение Коши для деформаций и закон Гука для компонент вектора $\boldsymbol{\omega}$, на $\partial \Omega$ будем иметь

$$
\begin{gathered}
\omega_{1}=\left\{\frac{1}{H_{2}} \frac{\partial u_{3}}{\partial a_{2}}-\frac{u_{2}}{H_{2} H_{3}} \frac{\partial H_{3}}{\partial a_{3}}\right\}-\frac{p_{2}}{2 \mu}, \quad \omega_{2}=\left\{\frac{u_{1}}{H_{1} H_{3}} \frac{\partial H_{1}}{\partial a_{2}}-\frac{1}{H_{1}} \frac{\partial H_{3}}{\partial a_{1}}\right\}+\frac{p_{1}}{2 \mu}, \\
\omega_{3}=\frac{1}{H_{1} H_{2}}\left\{\frac{\partial H_{2} u_{2}}{\partial a_{1}}-\frac{\partial H_{1} u_{1}}{\partial a_{2}}\right\} .
\end{gathered}
$$

Таким образом, вектор вращения $\boldsymbol{w}$ можно представить в виде

$$
2 \boldsymbol{w}=\boldsymbol{L}+\mu^{-1} \boldsymbol{D}
$$

где

$$
\begin{gathered}
L_{1}=\frac{2}{H_{2}} \frac{\partial u_{3}}{\partial a_{2}}-\frac{2 u_{2}}{H_{1} H_{3}} \frac{\partial H_{2}}{\partial a_{3}}, \quad L_{2}=\frac{2 u_{1}}{H_{1} H_{3}} \frac{\partial H_{1}}{\partial a_{3}}-\frac{2}{H_{1}} \frac{\partial u_{3}}{\partial a_{1}}, \\
L_{3}=\frac{2}{H_{1} H_{2}}\left\{\frac{\partial H_{2} u_{2}}{\partial a_{1}}-\frac{\partial H_{1} u_{1}}{\partial a_{2}}\right\} ; \quad D_{1}=-p_{2}, \quad D_{2}=-p_{1}, \quad D_{3}=0 .
\end{gathered}
$$


В декартовой системе координат для $\operatorname{rot} \boldsymbol{u}$ найдём:

$$
(\operatorname{rot} \boldsymbol{u})_{i}=2 \sum_{j=1}^{3} w_{j} \cos \left(a_{j}, x_{i}\right) l_{j}+\mu^{-1} d_{i},
$$

где

$$
l_{i}=\sum_{j=1}^{3} L_{j} \cos \left(a_{j}, x_{i}\right), \quad d_{i}=\sum_{j=1}^{3} D_{j} \cos \left(a_{j}, x_{i}\right) .
$$

Таким образом, для компонент $\boldsymbol{e}(\boldsymbol{r})$ имеем $e_{i}=-\mu l_{i}-d_{i}(i \equiv 1,2,3)$. С учётом введенных выше обозначений на $\partial \Omega$ можно записать

$$
\boldsymbol{F}(\boldsymbol{r})=\boldsymbol{q}^{(1)}(\boldsymbol{r}, \boldsymbol{u})+\mu \boldsymbol{q}^{(2)}(\boldsymbol{r}, \boldsymbol{p}),
$$

где $\boldsymbol{q}^{(1)}(\boldsymbol{r}, \boldsymbol{p})=\left\{p_{3},-d_{1},-d_{2},-d_{3}\right\}, \boldsymbol{q}^{(2)}(\boldsymbol{r}, \boldsymbol{u})=\left\{N_{1},-l_{1},-l_{2},-l_{3}\right\}$.

Таким образом, голоморфный вектор $\boldsymbol{F}(\boldsymbol{r})$ на $\partial \Omega$ может быть представлен в виде суммы двух четырёхкомпонентных векторов, зависящих соответственно только от вектора перемещения или только от вектора нагрузки.

Перейдём к решению задачи II. При задании граничных условий на поверхности $S_{u}$ будет задан четырёхкомпонентный вектор $\boldsymbol{q}^{(2)}(\boldsymbol{r}, \boldsymbol{u})$, на $S_{p}$ вектор $\boldsymbol{q}^{(1)}(\boldsymbol{r}, \boldsymbol{p})$. Тогда на $S_{u}$ и $S_{p}$ соответственно будут неизвестны значения четырёхкомпонентных векторов $\boldsymbol{q}^{(1)}(\boldsymbol{r}, \boldsymbol{p})$ и $\boldsymbol{q}^{(2)}(\boldsymbol{r}, \boldsymbol{u})$. С учётом этого систему для неизвестных можно представить в виде

$$
\begin{gathered}
-\frac{1}{2} \boldsymbol{F}(r)+\frac{1}{4 \pi} \iint_{S_{0}} \boldsymbol{M}\left(\left|\boldsymbol{r}-\boldsymbol{r}_{0}\right|\right) \boldsymbol{F}\left(\boldsymbol{r}_{0}\right) d S_{\boldsymbol{r}_{0}}+ \\
+\frac{1}{4 \pi} \iint_{S_{u}} \boldsymbol{M}\left(\left|\boldsymbol{r}-\boldsymbol{r}_{0}\right|\right) \boldsymbol{q}^{(1)}\left(\boldsymbol{r}_{0}, \boldsymbol{p}\right) d S_{\boldsymbol{r}_{0}}+\frac{\mu}{4 \pi} \iint_{S_{p}} \boldsymbol{M}\left(\left|\boldsymbol{r}-\boldsymbol{r}_{0}\right|\right) \boldsymbol{q}^{(2)}\left(\boldsymbol{r}_{0}, \boldsymbol{u}\right) d S_{\boldsymbol{r}_{0}}= \\
=-\frac{\mu}{4 \pi} \iint_{S_{u}} \boldsymbol{M}\left(\left|\boldsymbol{r}-\boldsymbol{r}_{0}\right|\right)\left\{N_{1},-l_{1},-l_{2},-l_{3}\right\}\left(\boldsymbol{r}_{0}, \boldsymbol{u}\right) d S_{\boldsymbol{r}_{0}}- \\
\quad-\frac{1}{4 \pi} \iint_{S_{p}} \boldsymbol{M}\left(\left|\boldsymbol{r}-\boldsymbol{r}_{0}\right|\right)\left\{p_{3},-d_{1},-d_{2},-d_{3}\right\}\left(\boldsymbol{r}_{0}, \boldsymbol{p}\right) d S_{\boldsymbol{r}_{0}-} \\
\quad-\frac{1}{4 \pi} \iint_{S_{u \sigma}} \boldsymbol{M}\left(\left|\boldsymbol{r}-\boldsymbol{r}_{0}\right|\right) \boldsymbol{F}^{*}\left(\boldsymbol{r}_{0}\right) d S_{\boldsymbol{r}_{0}}, \quad \boldsymbol{r} \in S_{0}, \quad(6)
\end{gathered}
$$

где $S_{0}=\partial \Omega-\left(S_{u} \cup S_{p} \cup S_{u \sigma}\right)$;

$$
\begin{gathered}
-\frac{1}{2} \boldsymbol{q}^{(1)}(\boldsymbol{r}, \boldsymbol{p})+\frac{1}{4 \pi} \iint_{S_{0}} \boldsymbol{M}\left(\left|\boldsymbol{r}-\boldsymbol{r}_{0}\right|\right) \boldsymbol{F}\left(\boldsymbol{r}_{0}\right) d S_{\boldsymbol{r}_{0}}+ \\
+\frac{1}{4 \pi} \iint_{S_{u}} \boldsymbol{M}\left(\left|\boldsymbol{r}-\boldsymbol{r}_{0}\right|\right) \boldsymbol{q}^{(1)}\left(\boldsymbol{r}_{0}, \boldsymbol{p}\right) d S_{\boldsymbol{r}_{0}}+\frac{\mu}{4 \pi} \iint_{S_{p}} \boldsymbol{M}\left(\left|\boldsymbol{r}-\boldsymbol{r}_{0}\right|\right) \boldsymbol{q}^{(2)}\left(\boldsymbol{r}_{0}, \boldsymbol{u}\right) d S_{\boldsymbol{r}_{0}}= \\
=-\frac{\mu}{4 \pi} \iint_{S_{u}} \boldsymbol{M}\left(\left|\boldsymbol{r}-\boldsymbol{r}_{0}\right|\right)\left\{N_{1},-l_{1},-l_{2},-l_{3}\right\}\left(\boldsymbol{r}_{0}, \boldsymbol{u}\right) d S_{\boldsymbol{r}_{0}}-
\end{gathered}
$$




$$
\begin{gathered}
-\frac{1}{4 \pi} \iint_{S_{p}} \boldsymbol{M}\left(\left|\boldsymbol{r}-\boldsymbol{r}_{0}\right|\right)\left\{p_{3},-d_{1},-d_{2},-d_{3}\right\}\left(\boldsymbol{r}_{0}, \boldsymbol{p}\right) d S_{\boldsymbol{r}_{0}}- \\
-\frac{1}{4 \pi} \iint_{S_{u \sigma}} \boldsymbol{M}\left(\left|\boldsymbol{r}-\boldsymbol{r}_{0}\right|\right) \boldsymbol{F}^{*}\left(\boldsymbol{r}_{0}\right) d S_{\boldsymbol{r}_{0}}, \quad \boldsymbol{r} \in S_{u} ; \quad(7) \\
-\frac{1}{2} \mu \boldsymbol{q}^{(2)}(\boldsymbol{r}, \boldsymbol{u})+\frac{1}{4 \pi} \iint_{S_{0}} \boldsymbol{M}\left(\left|\boldsymbol{r}-\boldsymbol{r}_{0}\right|\right) \boldsymbol{F}\left(\boldsymbol{r}_{0}\right) d S_{\boldsymbol{r}_{0}}+ \\
+\frac{1}{4 \pi} \iint_{S_{u}} \boldsymbol{M}\left(\left|\boldsymbol{r}-\boldsymbol{r}_{0}\right|\right) \boldsymbol{q}^{(1)}\left(\boldsymbol{r}_{0}, \boldsymbol{p}\right) d S_{\boldsymbol{r}_{0}}+\frac{\mu}{4 \pi} \iint_{S_{p}} \boldsymbol{M}\left(\left|\boldsymbol{r}-\boldsymbol{r}_{0}\right|\right) \boldsymbol{q}^{(2)}\left(\boldsymbol{r}_{0}, \boldsymbol{u}\right) d S_{\boldsymbol{r}_{0}}= \\
=-\frac{\mu}{4 \pi} \iint_{S_{u}} \boldsymbol{M}\left(\left|\boldsymbol{r}-\boldsymbol{r}_{0}\right|\right)\left\{N_{1},-l_{1},-l_{2},-l_{3}\right\}\left(\boldsymbol{r}_{0}, \boldsymbol{u}\right) d S_{\boldsymbol{r}_{0}}- \\
-\frac{1}{4 \pi} \iint_{S_{p}} \boldsymbol{M}\left(\left|\boldsymbol{r}-\boldsymbol{r}_{0}\right|\right)\left\{p_{3},-d_{1},-d_{2},-d_{3}\right\}\left(\boldsymbol{r}_{0}, \boldsymbol{p}\right) d S_{\boldsymbol{r}_{0}-} \\
-\frac{1}{4 \pi} \iint_{S_{u \sigma}} \boldsymbol{M}\left(\left|\boldsymbol{r}-\boldsymbol{r}_{0}\right|\right) \boldsymbol{F}^{*}\left(\boldsymbol{r}_{0}\right) d S_{\boldsymbol{r}_{0}}, \quad r \in S_{p} . \quad(8)
\end{gathered}
$$

Уравнения (6)-(8) являются сингулярными относительно $\boldsymbol{F}(\boldsymbol{r}), \boldsymbol{q}^{(1)}(\boldsymbol{r}, \boldsymbol{p})$, $\boldsymbol{q}^{(2)}(\boldsymbol{r}, \boldsymbol{u})$. Единственность решения (6)-(8) обеспечивается единственностью решения задачи $(\boldsymbol{u}, \boldsymbol{p})$, поскольку система (6)-(8) также позволяет восстанавливать голоморфный вектор по данным, которые заданны на части поверхности тела. После восстановления $\boldsymbol{F}(\boldsymbol{r}), \boldsymbol{q}^{(1)}(\boldsymbol{r}, \boldsymbol{p}), \boldsymbol{q}^{(2)}(\boldsymbol{r}, \boldsymbol{u})$ дальнейшее решение строится аналогично второму этапу задачи I, т. е. восстанавливается голоморфный вектор $\boldsymbol{B}(\boldsymbol{r})$ с учётом задания вектора $\boldsymbol{u}$ на $S_{u}$.

Очевидно, что задача II по отношению к задаче I является переопределённой. Так, решив задачу I на $S_{u}$ и $S_{p}$, можно восстановить соответственно векторы $\boldsymbol{u}$ и $\boldsymbol{p}$. Поэтому будем полагать, что граничные данные задачи II совпадут с определёнными из I, т. е. данные задачи II согласованы с решением задачи I. Последнее утверждение вытекает из постановки задач. Таким образом, по существу задача II и отличается от задачи I привлечением дополнительных данных для корректного решения задачи I.

Работа выполнена при поддержке РФФИ (код проекта 08-01-00168), фонда СО РАН ( проект № 72) и в рамках Программы поддержки ведущих научных школ (НШ-3066.2008.1.)

\section{БИБЛИОГРАФИЧЕСКИЙ СПИСОК}

1. Косарев Г. Л., Ризаев Э. З., Судо К. Определение параметров очагов землятресений по наблюдаемым остаточным смещениям земной поверхности // Докл. AH CCCP, 1986. Т. 288 , № 4 . - С. $75-81$.

2. Шваб А. А. Неклассическая упруго-пластическая задача // Изв. АН СССР. Мех. твёрд. тела, 1988. - № 1. - С. 140-146.

3. Шваб А. А. Некорректные статические задачи теории упругости // Изв. АН СССР. Мех. твёрд. тела, 1989. - №6. - С. 98-105.

4. Kubo S. Inverse Problems Related to the Mechanics and Fracture of Solids and Structures // JSME, Int. J. Ser. 1, 1988. - Vol. 31, No. 2. - P. 157-166.

5. Gao Z., Mura T. Elasticity problems with partially overspecified boundary conditions // International Journal of Engineering Science, 1991. - Vol. 29, No.6. - P. 685-692.

6. Tanaka M., Masuda Y. Boundary element method applied to some inverse problems // Engineering Analysis, 1986. - Vol. 3, No.3. - P. 138-143. 
7. Marin L., Háo D., Lesnic D. Conjugate Gradient-Boundary Element Method for the Cauchy Problem in Elasticity // Quart. J. Mech. Appl. Math., 2002. - Vol.55, No. 2. P. 227-247.

8. Marin L., Elliott L., Ingham D. B., Lesnic D. Boundary Element Regularisation Methods for Solving the Cauchy Problem in Linear Elasticity// Inverse Problems in Science and Engineering, 2002. - Vol. 10, No.4. - P. 335-337.

9. Marin L., Lesnic D. BEM first-order regularisation method in linear elasticity for boundary identification // Comput. Methets Appl. Mech. Engrg., 2003. - Vol.192, No.16-18. P. 2059-2071.

10. Schwab A. A. Nonclassical problems of deformed solid body / In: 8-Symposium, Vervormung und Bruch. - Magdeburg, 1988. - P. 90.

11. Fueter $R$. Über die analytische Darstellung der regulären Funktionen einer Quaternionenvariablen // Commentarii Mathematici Helvetici, 1935. - Vol. 8, No. 1. - P. 371-378.

12. Moisil Gr., Théodoresco N. Fonctions holomorphes dans l'espace// Mathematica, Cluj, 1931. - Vol. 5. - P. 142-159.

13. Бицадзе A. В. Основы теории аналитических функций. - М.: Наука, 1984. - 321 с.

14. Шваб А. А. Об одной локальной задаче продолжения / В сб.: Динамика сплошной среды. Bъи. 92. - Новосибирск: ИГиЛ СО АН СССР, 1989. - С. 23-28.

Поступила в редакцию 18/V/2009;

в окончательном варианте - 10/II/2010.

MSC: 35Q74

\section{BOUNDARY INTEGRAL EQUATION METHOD FOR HOLOMORPHIC VECTOR FOR PROBLEMS MONITORING ELASTICITY FIELD}

\section{A. A. Schwab}

M. A. Lavrentyev Institute of Hydrodynamics, Siberian Branch of RAS,

15, Lavrentyeva pr., Novosibirsk, 630090.

E-mail: schwab@ngs.ru

Consideration is given to a class of non-classical problems in elasticity theory which concerns the restoration of a full tensor of stresses in a body in the case where for one part of the body surface the loading vector and the displacement vector are prescribed, for other parts vector of displacements or vector of loads is known and for some parts of the body surface (its measure is not equal zero) the conditions are unknown. For solving of this problem boundary integral equations method for the holomorphic vector is proposed. It is shown that this method is efficient and can be applied to the nonclassical problems of mechanics.

Key words: boundary integral equation, non-classical problems in elasticity, monitoring problems, elasticity fields.

Original article submitted 18/V/2009; revision submitted 10/II/2010.

Albert A. Schwab (Dr. Sci. (Phys. \& Math.)), Leading Research Scientist, Lab. of Static Strength. 